\section{RSP}

http://www.rsp.fsp.usp.br/
Revista de Saúde Pública

\title{
A brief-review of the risk factors for covid-19 severity
}

\author{
J.E.Rod ${ }^{1,1,1, \mathrm{II}}$ (iD), Oscar Oviedo-Trespalacios ${ }^{\mathrm{II}, \mathrm{III}, \mathrm{IV}}$ (iD , Javier Cortes-Ramirez' \\ I Queensland University of Technology (QUT). School of Public Health and Social Work. Brisbane, \\ Queensland, Australia \\ " Queensland University of Technology (QUT). Institute of Health and Biomedical Innovation. Brisbane, \\ Queensland, Australia \\ III Queensland University of Technology (QUT). Centre for Accident Research and Road Safety Queensland \\ (CARRS-Q). Brisbane, Queensland, Australia \\ ıv Universidad del Norte. Departamento de ingeniería. Barranquila, Atlántico, Colombia
}

\begin{abstract}
The World Health Organization has emphasized that one of the most important questions to address regarding the covid-19 pandemic is to understand risk factors for disease severity. We conducted a brief review that synthesizes the available evidence and provides a judgment on the consistency of the association between risk factors and a composite end-point of severe-fatal covid-19. Additionally, we also conducted a comparability analysis of risk factors across 17 studies. We found evidence supporting a total of 60 predictors for disease severity, of which seven were deemed of high consistency, 40 of medium and 13 of low. Among the factors with high consistency of association, we found age, C-reactive protein, D-dimer, albumin, body temperature, SOFA score and diabetes. The results suggest that diabetes might be the most consistent comorbidity predicting disease severity and that future research should carefully consider the comparability of reporting cases, factors, and outcomes along the different stages of the natural history of covid-19.
\end{abstract}

DESCRIPTORS: Coronavirus Infections, epidemiology. Fatal Outcome. Risk Factors. Review.

\footnotetext{
Correspondence:

CARRS-Q, K Block,

130 Victoria Park Road, 4059

Kelvin Grove,

Brisbane, QLD, Australia.

E-mail: j.rodriguezzabala@qut.edu.au

Received: Mar 25, 2020

Approved: Apr 14, 2020

How to cite: Rod JE. Oviedo-

Trespalacios O, Cortes-Ramirez J.

A brief-review of the risk factors

for covid-19 severity. Rev Saude

Publica. 2020;54:60.

Copyright: This is an open-access article distributed under the terms of the Creative Commons Attribution License, which permits unrestricted use, distribution, and reproduction in any medium, provided that the original author and source are credited.
} 


\section{INTRODUCTION}

More than 200 countries and territories have reported confirmed cases of the novel coronavirus disease covid-19, characterized as a pandemic by the World Health Organisation on April 7, 20201. As this global health emergency tests the resilience of health systems around the world, health care and public health practitioners are required to have high quality evidence to identify its most significant risks and prioritize resources where they are most needed.

One of the most important questions to address the currently unfolding pandemic is "what are the risk factors for severe illness or death?"1,2. Systematic reviews and meta-analysis paired with a standardised method to assess the quality of evidence are deemed to provide the best evidence by current standards ${ }^{3}$. For example, the GRADE evidence assessment focuses on comparing factors or outcomes across studies to provide an evidence synthesis. If researchers conduct a GRADE evaluation for covid-19 severity in the future, the time investment and internal validity of the assessment will be heavily influenced by the consistency of categorizations and reporting of cases, factors, and outcomes across studies.

Since scientific publications addressing the pandemic are being produced rapidly, including risk factor studies, summarizing and sharing such information is of paramount importance to support an efficient and rapid response. An early review of risk factor studies could also provide some insight on the undesirable heterogeneity of definitions and reporting that might affect later evidence evaluations. Therefore, we compiled a brief summary of the literature evaluating the risk factors for covid-19 disease severity with a two-fold purpose: (i) to provide healthcare and public health professionals with a reference list of the consistency of risk factors for covid-19 severity, and (ii) to inform researchers about the consistency of reporting in the available literature.

\section{METHODS}

We conducted a review to assess studies looking for risk factors of severity or death for covid-19, using a composite outcome of disease severity-fatality (CSF) ${ }^{4}$. Unstructured searches using the terms: "disease attributes," "clinical findings," "clinical features," "clinical characteristic," "novel coronavirus," "covid-19," "SARS-Cov-2," "fatality," "fatal," "death," "mortality," "severity," "disease severity," "predictor," and "risk factor" were performed to identify articles written in English available on PubMed, Scopus and MedRxiv. Articles were selected for the review if they included a comparison between non-severe and CSF cases according to the categorization of severity in each article. After completing article selection, we assessed the consistence of statistically significant associations for a particular risk factor by classifying it as high, medium, and low, following two criteria: (i) a positive difference in the total number of studies reporting statistical significance dissimilarities between non-severe and CSF, minus the number of studies without statistical significance for the same factor, and, (ii) the reporting of statistically significant estimates when performing multivariate statistics. The consistency of association was categorized as high (both criteria were met), medium (one criterion), low (none). Additionally, we assessed heterogeneity by comparing the terminology, units, statistical descriptions, and cut-off points of each risk factor and reported the highest comparable (hc) number across studies. We then subtracted the total of studies including the risk factor by the hc number across the sample (Table).

\section{RESULTS}

We identified a total of 17 studies, with most of them relying on a retrospective cross-sectional design and reporting data using descriptive statistics. Only three studies 
Table. Summary of risk factors associated with covid-19 severity and evaluation of reporting consistency

\begin{tabular}{|c|c|c|c|c|c|c|c|c|}
\hline \multirow[b]{2}{*}{$\begin{array}{l}\text { RISK } \\
\text { FACTORS }\end{array}$} & \multicolumn{3}{|c|}{ Consistency computation for each factor } & \multirow[b]{2}{*}{$\begin{array}{l}\text { Studies not } \\
\text { reporting } p \\
\text { values }(n)\end{array}$} & \multirow[b]{2}{*}{$\begin{array}{c}\text { Total } \\
(p<0.05) \\
- \text { total } \\
(p>0.05)\end{array}$} & \multirow[b]{2}{*}{$\begin{array}{c}\text { Results of } \\
(\boldsymbol{p}<0.05) \\
\text { multivariate } \\
\text { statistics }\end{array}$} & \multirow[b]{2}{*}{$\begin{array}{l}\text { Consistency } \\
\\
\text { œ• High } \\
\bullet \circ \text { Medium } \\
\bullet \circ \circ \text { Low }\end{array}$} & \multirow[b]{2}{*}{$\begin{array}{c}\text { Heterogeneity } \\
\text { hc } \# \\
\text { (Total - hc } \# / \text { total) } \\
\text { hc-statistic } \\
\text { hc-definition, unit }\end{array}$} \\
\hline & $\begin{array}{c}\text { All studies ( } n) \\
\text { with } p<0.05 \text { for } \\
\text { differences } \\
\text { non-severe vs } \\
\text { CSF }\end{array}$ & $\begin{array}{c}\% \text { of } \\
p<0.05 \text { studies } \\
\text { supporting a } \\
\text { value direction } \\
\text { or the presence } \\
\text { of the risk factor }\end{array}$ & $\begin{array}{c}\text { Studies } \\
p>0.05(n)\end{array}$ & & & & & \\
\hline Age & $\begin{array}{c}\text { Zhang et al. } .^{6}(19) \\
\text { Wang et al. } \\
\text { Tian et al. }{ }^{8}(262) \\
\text { Liu et al. } .^{9}(109) \\
\text { Liu et al. } .^{10}(78) \\
\text { Li et al. }{ }^{11}(84) \\
\text { Caramelo et al. }{ }^{12} \\
(72314) \\
\text { Tang et al. } .^{13}(183) \\
\text { Ruan et al. }{ }^{14}(150) \\
\text { Zhou et al. }{ }^{15}(191) \\
\text { Wu et al. } .^{16}(201)\end{array}$ & $100 \%-\uparrow$ & $\begin{array}{c}\text { Huang et al. }{ }^{17} \\
(41)\end{array}$ & $\begin{array}{c}\text { Guan et al. } .^{18} \\
(1099) \\
\text { Yang et al. } .^{19}(52) \\
\text { Young et al. }{ }^{20}(18) \\
\text { Ji et al. } .^{21}(\mathrm{NA}) \\
\text { Zhonghua }^{22} \\
(44672)\end{array}$ & 10 & $\begin{array}{c}\text { Liu et al. } .^{10} \\
\geq 60: \text { OR } 8.5 \\
95 \% \text { Cl } 1.6-44.8 \\
\\
\text { Caramelo et al. }{ }^{12} \\
\text { 50-59: OR } 6.7 \\
95 \% \text { Cl } 2.9-15.2 \\
\text { } 80 \text { : OR } 86.6 \\
\text { 95\% Cl } 32.6- \\
202.4 \\
\text { Zhou et al. }{ }^{15} \\
\text { NA: OR } 1.1 \\
\text { 95\% Cl 1.0 - } 1.2\end{array}$ & $\bullet \bullet \bullet$ & $\begin{array}{c}12 \\
(5 / 17)=29 \% \\
\text { Median (IQR) } \\
\text { Age, years }\end{array}$ \\
\hline $\begin{array}{l}\text { C- reactive } \\
\text { protein }\end{array}$ & $\begin{array}{c}\text { Zhang et al. }{ }^{6}(19) \\
\text { Li et al. } .^{11}(84) \\
\text { Liu et al. } .^{10}(78) \\
\text { Liu et al. } .^{9}(109) \\
\text { Ruan et al. }{ }^{14}(150) \\
\text { Wu et al. } .^{16}(201)\end{array}$ & $100 \%-\uparrow$ & 0 & $\begin{array}{l}\text { Young et al. } .^{20}(18) \\
\text { Guan et al. } .^{18} \\
(1099)\end{array}$ & 6 & $\begin{array}{c}\text { Liu et al. } .^{10} \\
>8.2 \mathrm{mg} / \mathrm{L}: \text { OR } \\
10.5 \\
95 \% \mathrm{Cl} 1.2-34.7\end{array}$ & $\bullet \bullet \bullet$ & $\begin{array}{c}6 \\
\begin{array}{c}(2 / 8) \\
=\end{array} 25 \% \\
\text { Median (IQR) } \\
\text { C-reactive } \\
\text { protein, mg/L }\end{array}$ \\
\hline D- Dimer & $\begin{array}{c}\text { Tang et al. }{ }^{13}(183) \\
\text { Huang et al. }{ }^{17}(41) \\
\text { Zhang et al. } .^{6}(19) \\
\text { Wang et al. } \\
\text { Liu et al. } .^{9}(109) \\
\text { Zhou et al.15 } 1(191) \\
\text { Wu et al. } .^{16}(201)\end{array}$ & $100 \%-\uparrow$ & Liu et al. ${ }^{10}(78)$ & $\begin{array}{c}\text { Guan et al. }{ }^{18} \\
\text { (1099) }\end{array}$ & 6 & $\begin{array}{c}\text { Zhou et al. }{ }^{15} \\
>1 \mu \mathrm{g} / \mathrm{mL}: \text { OR } \\
18.42 \\
95 \% \mathrm{Cl} 2.6-128.5\end{array}$ & $\bullet \bullet$ & $\begin{array}{c}7 \\
(2 / 9)=22 \% \\
\text { Median (IQR) } \\
\text { D-dimer, } \mu \mathrm{g} / \mathrm{mL}\end{array}$ \\
\hline Albumin & $\begin{array}{c}\text { Liu et al. } .^{10}(78) \\
\text { Huang et al. }{ }^{17}(41) \\
\text { Ruan et al. }{ }^{14}(150) \\
\text { Zhou et al.15 (191) } \\
\text { Wu et al. } .^{16}(201)\end{array}$ & $100 \%-\downarrow$ & 0 & 0 & 5 & $\begin{array}{c}\text { Liu et al. }{ }^{10} \\
<40 \mathrm{~g} / \mathrm{L}: \text { OR } 7.4 \\
95 \% \mathrm{Cl} 1.1-50.0\end{array}$ & $\bullet \bullet \bullet$ & $\begin{array}{c}4 \\
(1 / 5)=20 \% \\
\text { Median (IQR) } \\
\text { Albumin, g/L }\end{array}$ \\
\hline $\begin{array}{l}\text { Body } \\
\text { temperature }\end{array}$ & $\begin{array}{l}\text { Huang et al. }{ }^{17}(41) \\
\text { Li et al. }{ }^{11}(84) \\
\text { Liu et al. } .^{10}(78) \\
\text { Wu et al. } .^{16}(201)\end{array}$ & $75 \%-\uparrow$ & Tian et al. ${ }^{8}(262)$ & $\begin{array}{c}\text { Guan et al. } .^{18} \\
(1099) \\
\text { Young et al. } .^{20}(18)\end{array}$ & 3 & $\begin{array}{c}\text { Liu et al. } .^{10} \\
\geq 37.3^{\circ}: \text { OR } 8.9 \\
95 \% \mathrm{Cl} 1.0-78.1\end{array}$ & $\bullet \bullet$ & $\begin{array}{c}4 \\
(3 / 7)=43 \% \\
\\
\text { Median (IQR) } \\
\text { Highest } \\
\text { temperature, C }\end{array}$ \\
\hline $\begin{array}{l}\text { SOFA } \\
\text { score }\end{array}$ & $\begin{array}{c}\text { Liu et al. } .^{9}(109) \\
\text { Zhou et al. }{ }^{15}(191)\end{array}$ & $100 \%-\uparrow$ & 0 & 0 & 2 & $\begin{array}{c}\text { Zhou et al. }{ }^{15} \\
\text { NA: OR } 5.7 \\
95 \% \mathrm{Cl} 2.6-12.2\end{array}$ & $\bullet \bullet \bullet$ & $\begin{array}{c}2 \\
(0 / 2)=0 \% \\
\text { Median (IQR) } \\
\text { SOFA score, NA }\end{array}$ \\
\hline Diabetes & $\begin{array}{c}\text { Caramelo et al. }{ }^{12} \\
\quad(72314) \\
\text { Li et al. }{ }^{11}(84) \\
\text { Wang et al. } .^{7}(138) \\
\text { Liu et al. } .^{9}(109) \\
\text { Zhou et al. }{ }^{15}(191) \\
\text { Wu et al. } .^{16}(201)\end{array}$ & $\begin{array}{c}100 \% \text { - } \\
\text { Presence }\end{array}$ & $\begin{array}{l}\text { Huang et al. } .^{17}(41) \\
\text { Zhang et al. } .^{6}(19) \\
\text { Liu et al. }{ }^{10}(78) \\
\text { Ruan et al. } .^{14}(150)\end{array}$ & $\begin{array}{c}\text { Guan et al. }{ }^{18} \\
(1099) \\
\text { Zhonghua }{ }^{22} \\
(44672) \\
\text { Yang et al. } .^{19}(52)\end{array}$ & 2 & $\begin{array}{l}\text { Caramelo et al. }{ }^{12} \\
\text { Diabetes: OR } 9.0 \\
95 \% \text { Cl } 7.4-11.3\end{array}$ & $\bullet \bullet$ & $\begin{array}{c}13 \\
(0 / 13)=0 \% \\
\text { Percentages } \\
\text { Diabetes, NA }\end{array}$ \\
\hline $\begin{array}{l}\text { Lymphocyte } \\
\text { count }\end{array}$ & $\begin{array}{l}\text { Zhang et al. } .^{6}(19) \\
\text { Li et al. }{ }^{11}(84) \\
\text { Wang et al. } .^{7}(138) \\
\text { Huang et al. }{ }^{17}(41) \\
\text { Liu et al. } .^{9}(109) \\
\text { Ruan et al. } .^{14}(150) \\
\text { Wu et al. } .^{16}(201) \\
\text { Zhou et al. }{ }^{15}(191)\end{array}$ & $100 \%-\downarrow$ & $\begin{array}{l}\text { Yang et al. } .^{19}(52) \\
\text { Liu et al. } .^{10}(78)\end{array}$ & $\begin{array}{l}\text { Young et al. } .^{20}(18) \\
\text { Guan et al. } .^{18} \\
\text { (1099) }\end{array}$ & 6 & NA & $\bullet \bullet \circ$ & $\begin{array}{c}9 \\
(3 / 12)=25 \% \\
\text { Median (IQR) } \\
\text { Lymphocyte } \\
\text { count, } \times 109 / \text { L }\end{array}$ \\
\hline
\end{tabular}


Table. Summary of risk factors associated with covid-19 severity and evaluation of reporting consistency. Continuation

\begin{tabular}{|c|c|c|c|c|c|c|c|c|}
\hline Dyspnea & $\begin{array}{l}\text { Li et al. }{ }^{11}(84) \\
\text { Huang et al. } .^{17}(41) \\
\text { Tian et al. }{ }^{8}(262) \\
\text { Wang et al. }{ }^{7}(138) \\
\text { Ruan et al. }{ }^{14}(150) \\
\text { Wu et al. } .^{16}(201)\end{array}$ & $\begin{array}{l}100 \%- \\
\text { Presence }\end{array}$ & Zhang et al. ${ }^{6}(19)$ & $\begin{array}{c}\text { Yang et al. }{ }^{19}(52) \\
\text { Guan et al. }{ }^{18} \\
\text { (1099) } \\
\text { Young et al. } .^{20}(18)\end{array}$ & 5 & NA & $\bullet \bullet \circ$ & $\begin{array}{c}10 \\
(0 / 10)=0 \% \\
\text { Percentages } \\
\text { Dyspnea, NA }\end{array}$ \\
\hline $\begin{array}{l}\text { White blood } \\
\text { Cell count }\end{array}$ & $\begin{array}{l}\text { Huang et al. } .^{17}(41) \\
\text { Wang et al. }{ }^{7}(138) \\
\text { Liu et al. } .^{9}(109) \\
\text { Ruan et al. }{ }^{14}(150) \\
\text { Zhou et al. }{ }^{15}(191) \\
\text { Wu et al. } .^{16}(201) \\
\text { Zhang et al. } .^{6}(19)\end{array}$ & $100 \%-\uparrow$ & $\begin{array}{l}\text { Li et al. } .^{11}(84) \\
\text { Liu et al. }{ }^{10}(78)\end{array}$ & $\begin{array}{c}\text { Guan et al. }{ }^{18} \\
\quad(1099) \\
\text { Young et al. } .^{20}(18)\end{array}$ & 5 & NA & $\bullet \bullet ०$ & $\begin{array}{c}8 \\
(3 / 11)=27 \% \\
\text { Median (IQR) } \\
\text { WBC count, } \\
\times 109 / \mathrm{L}\end{array}$ \\
\hline Procalcitonin & $\begin{array}{c}\text { Li et al. }{ }^{11}(84) \\
\text { Wang et al. } .^{7}(138) \\
\text { Huang et al. } .^{17}(41) \\
\text { Liu et al. } .^{9}(109) \\
\text { Zhou et al. } .^{15}(191)\end{array}$ & $100 \%-\uparrow$ & Liu et al. ${ }^{10}(78)$ & $\begin{array}{c}\text { Guan et al. }{ }^{18} \\
(1099)\end{array}$ & 4 & NA & $\bullet \bullet$ & $\begin{array}{c}6 \\
(1 / 7)=14 \% \\
\text { Median (IQR) } \\
\text { Procalcitonin, } \\
\text { ng/ml }\end{array}$ \\
\hline $\begin{array}{l}\text { Lactate } \\
\text { dehydrogenase }\end{array}$ & $\begin{array}{l}\text { Wang et al. }{ }^{7}(138) \\
\text { Huang et al. } .^{17}(41) \\
\text { Liu et al. } .^{9}(109) \\
\text { Zhou et al. }{ }^{15}(191) \\
\text { Wu et al. } .^{16}(201)\end{array}$ & $100 \%-\uparrow$ & Ruan et al. ${ }^{14}$ (150) & $\begin{array}{c}\text { Young et al. } .^{20}(18) \\
\text { Guan et al. } .^{18} \\
(1099)\end{array}$ & 4 & $\mathrm{NA}$ & $\bullet \bullet ०$ & $\begin{array}{c}7 \\
(1 / 8)=13 \% \\
\text { Median (IQR) } \\
\text { LDH, U/L }\end{array}$ \\
\hline $\begin{array}{l}\text { Cardiac } \\
\text { troponins }\end{array}$ & $\begin{array}{l}\text { Wang et al. }{ }^{7}(138) \\
\text { Huang et al. } .^{17}(41) \\
\text { Ruan et al. }{ }^{14}(150) \\
\text { Zhou et al. }{ }^{15}(191)\end{array}$ & $100 \%-\uparrow$ & 0 & 0 & 4 & NA & $\bullet \bullet \circ$ & $\begin{array}{c}3 \\
(1 / 4)=25 \% \\
\text { Median (IQR) } \\
\text { Hypersensitive } \\
\text { troponin I, pg/ml }\end{array}$ \\
\hline $\begin{array}{l}\text { Prothrombin } \\
\text { time }\end{array}$ & $\begin{array}{l}\text { Huang et al. } .^{17}(41) \\
\text { Tang et al. } .^{13}(183) \\
\text { Zhou et al. }{ }^{15}(191) \\
\text { Wu et al. } .^{16}(201)\end{array}$ & $100 \%-\uparrow$ & Wang et al. ${ }^{7}(138)$ & Yang et al. ${ }^{19}$ (52) & 3 & $\mathrm{NA}$ & $\bullet \bullet ०$ & $\begin{array}{c}5 \\
(1 / 6)=17 \% \\
\text { Median (IQR) } \\
\text { Prothrombin } \\
\quad \text { time, s, }\end{array}$ \\
\hline $\begin{array}{l}\text { Blood urea } \\
\text { nitrogen }\end{array}$ & $\begin{array}{l}\text { Ruan et al. } .^{14}(150) \\
\text { Liu et al. }{ }^{9}(109) \\
\text { Wang et al. }{ }^{7}(138)\end{array}$ & $100 \%-\uparrow$ & 0 & 0 & 3 & NA & $\bullet \bullet ०$ & $\begin{array}{c}3 \\
(0 / 3)=0 \% \\
\text { Blood urea } \\
\text { nitrogen, } \\
\text { mmol/L }\end{array}$ \\
\hline Total bilirubin & $\begin{array}{l}\text { Ruan et al. } .^{14}(150) \\
\text { Wang et al. }{ }^{7}(138) \\
\text { Huang et al. } .^{17}(41) \\
\text { Wu et al. } .^{16}(201)\end{array}$ & $100 \%-\uparrow$ & Liu et al. ${ }^{9}(109)$ & $\begin{array}{c}\text { Yang et al. }{ }^{19}(52) \\
\text { Guan et al. }{ }^{18} \\
(1099)\end{array}$ & 3 & NA & $\bullet \bullet \circ$ & $\begin{array}{c}4 \\
(3 / 7)=43 \% \\
\text { Total bilirubin, } \\
\mu \mathrm{mol} / \mathrm{L}\end{array}$ \\
\hline Interleukin-6 & $\begin{array}{l}\text { Ruan et al. }{ }^{14}(150) \\
\text { Zhou et al. }{ }^{15}(191) \\
\text { Wu et al. } .^{16}(201)\end{array}$ & $100 \%-\uparrow$ & 0 & 0 & 3 & NA & $\bullet \bullet \circ$ & $\begin{array}{c}3 \\
(0 / 3)=0 \% \\
\text { Median (IQR) } \\
\text { IL-6, pg/mL }\end{array}$ \\
\hline Serum ferritin & $\begin{array}{l}\text { Ruan et al. }{ }^{14}(150) \\
\text { Zhou et al. }{ }^{15}(191) \\
\text { Wu et al. }{ }^{16}(201)\end{array}$ & $100 \%-\uparrow$ & 0 & 0 & 3 & NA & $\bullet \bullet ०$ & $\begin{array}{c}3 \\
(0 / 3)=0 \% \\
\text { Median (IQR) } \\
\text { Serum ferritin, } \\
\mathrm{ng} / \mathrm{mL}\end{array}$ \\
\hline Comorbidity & $\begin{array}{l}\text { Li et al. }{ }^{11}(84) \\
\text { Wang et al. }{ }^{7}(138) \\
\text { Zhang et al. }{ }^{6}(19) \\
\text { Ruan et al. }{ }^{14}(150) \\
\text { Zhou et al. }{ }^{15}(191)\end{array}$ & $\begin{array}{l}100 \% \text { - } \\
\text { Presence }\end{array}$ & $\begin{array}{l}\text { Huang et al. }{ }^{17}(41) \\
\text { Tang et al. }{ }^{13}(183)\end{array}$ & $\begin{array}{c}\text { Guan et al. }{ }^{18} \\
(1099) \\
\text { Yang et al. }{ }^{19}(52)\end{array}$ & 3 & NA & $\bullet \bullet ०$ & $\begin{array}{c}8 \\
(0 / 8)=0 \% \\
\text { Percentages } \\
\text { Comorbidity, NA }\end{array}$ \\
\hline $\begin{array}{l}\text { Neutrophil } \\
\text { count }\end{array}$ & $\begin{array}{l}\text { Huang et al. } .^{17}(41) \\
\text { Wang et al. }{ }^{7}(138) \\
\text { Liu et al. } .^{9}(109) \\
\text { Wu et al. } .^{16}(201)\end{array}$ & $75 \%-\uparrow$ & $\begin{array}{l}\text { Liu et al. }{ }^{10}(78) \\
\text { Li et al. } .^{11}(84)\end{array}$ & Young et al..$^{20}(18)$ & 2 & $\mathrm{NA}$ & $\bullet \bullet \circ$ & $\begin{array}{c}7 \\
(0 / 7)=0 \% \\
\text { Median (IQR) } \\
\text { Neutrophil count, } \\
\times 109 / \mathrm{L}\end{array}$ \\
\hline
\end{tabular}


Table. Summary of risk factors associated with covid-19 severity and evaluation of reporting consistency. Continuation

\begin{tabular}{ll}
\hline Creatine & Wang et al. ${ }^{7}(138)$ \\
kinase MB & Zhou et al. ${ }^{15}(191)$
\end{tabular}

Winase $M B$ et al. ${ }^{16}(201)$

$100 \%-\uparrow \quad$ Liu et al. ${ }^{9}(109)$

0

$(0 / 4)=0 \%$

CURB-65

Liu et al..$^{9}(109)$

$100 \%-\uparrow$

0

0

NA

CK-MB, U/L

2

$(0 / 2)=0 \%$

\begin{tabular}{|c|c|c|c|c|c|}
\hline $\begin{array}{l}\text { Respiratory } \\
\text { rate }\end{array}$ & $\begin{array}{c}\text { Huang et al. }{ }^{17}(41) \\
\text { Liu et al. } .^{10}(78) \\
\text { Tian et al. }{ }^{8}(262) \\
\text { Zhou et al. } .^{15}(191)\end{array}$ & $100 \%-\uparrow$ & $\begin{array}{c}\text { Li et al. }{ }^{11}(84) \\
\text { Wang et al. } .^{7}(138)\end{array}$ & Young et al. ${ }^{20}(18)$ & 2 \\
\hline
\end{tabular}

Zhou et al. ${ }^{15}(191)$

\section{Lymphocyte}

ratio

Li et al. ${ }^{11}(84)$

$100 \%-\downarrow$

0

0

NA

$100 \%$ - $\quad$ Ruan et al. ${ }^{14}(150)$
Presence

0

NA

Median (IQR)

CURB-65 score, NA 3

$(4 / 7)=57 \%$

Median (IQR)

Respiratory rate,

breaths*min

1
$(0 / 1)=0 \%$

Chronic Liu et al. ${ }^{9}$ (109)

Presence

NA

Mean (SD)

Lymphocyte ratio, \%

3

$(0 / 3)=0 \%$

Percentages

Chronic kidney

dis ease, NA

2

$(0 / 2)=0 \%$

Chest pain $\quad$ Li et al. ${ }^{11}(84) \quad 100 \%$ -

$0 \quad$ Yang et al. ${ }^{19}(52)$

NA

Percentages

Chest pain, NA

$$
(0 / 1)=0 \%
$$

Neutrophil

ratio

Li et al. ${ }^{11}(84) \quad 100 \%-\uparrow$

0

0

NA

Percentage

Neutrophil ratio $\%$

1

$(0 / 1)=0 \%$

Fibrinogen

degradation Tang et al. ${ }^{13}(183) \quad 100 \%-\uparrow$

0

0

NA

Median (IQR) FDP, ug/mL

1

$(0 / 1)=0 \%$

Myoglobin $\quad$ Ruan et al. ${ }^{14}(150) \quad 100 \%-\uparrow$

0

NA

Mean (SD)

Myoglobin, ng/ $\mathrm{mL}$

1

$(0 / 1)=0 \%$

APACHE II Liu et al. ${ }^{9}(109) \quad 100 \%-\uparrow$

0

0

NA

Median (IQR)

APACHE II score, NA

1

$(0 / 1)=0 \%$

PaO2:FiO2

Liu et al. ${ }^{9}(109)$

$100 \%-\downarrow$

0

0

1

NA

Median (IQR)

$\mathrm{PaO} 2: \mathrm{FiO} 2$,

$\mathrm{mmHg}$

$(0 / 1)=0 \%$

Globulin

Wu et al. ${ }^{16}(201)$

$100 \%-\uparrow$

0

0

1

NA

Median (IQR)

Globulin, g/L

Continue 
Table. Summary of risk factors associated with covid-19 severity and evaluation of reporting consistency. Continuation

\begin{tabular}{|c|c|c|c|c|c|c|c|c|}
\hline Prealbumin & Wu et al. ${ }^{16}(201)$ & $100 \%-\downarrow$ & 0 & 0 & 1 & $\mathrm{NA}$ & $\bullet \bullet ०$ & $\begin{array}{c}1 \\
(0 / 1)=0 \% \\
\text { Median }(\mathrm{IQR}) \\
\text { prealbumin, } \mathrm{mg} / \mathrm{L}\end{array}$ \\
\hline Urea & Wu et al. ${ }^{16}(201)$ & $100 \%-\uparrow$ & 0 & 0 & 1 & NA & $\bullet \bullet$ & $\begin{array}{c}1 \\
(0 / 1)=0 \% \\
\text { Median (IQR) } \\
\text { prealbumin, mM }\end{array}$ \\
\hline Glucose & Wu et al. ${ }^{16}(201)$ & $100 \%-\uparrow$ & 0 & 0 & 1 & NA & $\bullet \bullet$ & $\begin{array}{c}1 \\
(0 / 1)=0 \% \\
\text { Median (IQR) } \\
\text { Glucose, mM }\end{array}$ \\
\hline Cholinesterase & Wu et al. ${ }^{16}(201)$ & $100 \%-\otimes$ & 0 & 0 & 1 & $\mathrm{NA}$ & $\bullet \bullet$ & $\begin{array}{c}1 \\
(0 / 1)=0 \% \\
\text { Median (IQR) } \\
\text { Cholinesterase, } \\
\text { U/L }\end{array}$ \\
\hline Cystatin C & Wu et al. ${ }^{16}(201)$ & $100 \%-\uparrow$ & 0 & 0 & 1 & $\mathrm{NA}$ & $\bullet \bullet ०$ & $\begin{array}{c}1 \\
(0 / 1)=0 \% \\
\text { Median (IQR) } \\
\text { Cystatin C, mg/L }\end{array}$ \\
\hline$\alpha-\mathrm{HBDH}$ & Wu et al..$^{16}(201)$ & $100 \%-\uparrow$ & 0 & 0 & 1 & $\mathrm{NA}$ & $\bullet \bullet$ & $\begin{array}{c}1 \\
(0 / 1)=0 \% \\
\text { Median (IQR) } \\
\alpha-\mathrm{HBDH} 100 \\
\mathrm{U} / \mathrm{L}\end{array}$ \\
\hline LDL & Wu et al..$^{16}(201)$ & $100 \%-\downarrow$ & 0 & 0 & 1 & NA & $\bullet \bullet$ & $\begin{array}{c}1 \\
(0 / 1)=0 \% \\
\text { Median }(I Q R) \\
\text { LDL, } \mathrm{mM}\end{array}$ \\
\hline Heart Rate & Zhou et al. ${ }^{15}$ (191) & $100 \%-\uparrow$ & 0 & 0 & 1 & $\mathrm{NA}$ & $\bullet \bullet$ & $\begin{array}{c}1 \\
(0 / 1)=0 \% \\
\text { Percentages } \\
\text { Heart Rate } \geq 125, \\
\text { beats } / \mathrm{min}\end{array}$ \\
\hline $\begin{array}{l}\text { Health system } \\
\text { burden in } \\
\text { Hubei }\end{array}$ & Ji et al..$^{21}$ (NA) & $100 \%-\uparrow$ & 0 & 0 & 1 & NA & $\bullet \bullet$ & $\begin{array}{c}1 \\
(0 / 1)=0 \% \\
\text { NA } \\
\text { NA }\end{array}$ \\
\hline $\begin{array}{l}\text { Days from } \\
\text { onset of } \\
\text { symptoms to } \\
\text { hospital }\end{array}$ & $\begin{array}{l}\text { Li et al. }{ }^{11}(84) \\
\text { Wang et al. }{ }^{7}(138) \\
\text { Tian et al. } .^{8}(262)^{*}\end{array}$ & $100 \%-\uparrow$ & $\begin{array}{l}\text { Zhang et al. }{ }^{6}(19) \\
\text { Zhou et al. }{ }^{15}(191)\end{array}$ & Huang et al. ${ }^{17}(41)$ & 1 & NA & $\bullet \bullet ०$ & $\begin{array}{c}5 \\
(1 / 6)=17 \% \\
\text { Median (IQR) } \\
\text { Symptom onset } \\
\text { to admission, } \\
\text { days }\end{array}$ \\
\hline O2 saturation & Li et al..$^{11}(84)$ & $100 \%-\downarrow$ & Liu et al..$^{10}(78)$ & Young et al. ${ }^{20}(18)$ & 0 & NA & $\bullet \bullet$ & $\begin{array}{c}3 \\
(0 / 3)=0 \% \\
\text { Median (IQR) } \\
\text { Oxygen } \\
\text { saturation, \% }\end{array}$ \\
\hline Fibrinogen & Liu et al..$^{9}(109)$ & $100 \%-\uparrow$ & Tang et al..$^{13}(183)$ & 0 & 0 & NA & $\bullet \bullet$ & $\begin{array}{c}2 \\
(0 / 2)=0 \% \\
\text { Median }(\mathrm{IQR}) \\
\text { Fibrinogen, } \\
\mathrm{g} / \mathrm{L}\end{array}$ \\
\hline
\end{tabular}

Continue 
Table. Summary of risk factors associated with covid-19 severity and evaluation of reporting consistency. Continuation

\begin{tabular}{|c|c|c|c|c|c|c|c|c|}
\hline Smoking & Liu et al. ${ }^{10}(78)$ & $\begin{array}{l}100 \% \text { - } \\
\text { Presence }\end{array}$ & $\begin{array}{l}\text { Huang et al. }{ }^{17}(41) \\
\text { Zhang et al. }{ }^{6}(19) \\
\text { Zhou et al. }{ }^{15}(191)\end{array}$ & $\begin{array}{c}\text { Guan et al. }{ }^{18} \\
\text { (1099) } \\
\text { Yang et al. }{ }^{19}(52)\end{array}$ & -2 & $\begin{array}{c}\text { Liu et al. }{ }^{10} \\
\text { Past use: OR } 14.3 \\
95 \% \text { Cl } 1.6-25.0\end{array}$ & $\bullet \bullet$ & $\begin{array}{c}5 \\
(1 / 6=17 \%) \\
\text { Percentages } \\
\text { Current smoker, } \\
\text { NA }\end{array}$ \\
\hline $\begin{array}{l}\text { Chronic } \\
\text { respiratory } \\
\text { disease }\end{array}$ & $\begin{array}{c}\text { Caramelo et al. }{ }^{12} \\
(72314) \\
\text { Li et al. }{ }^{11}(84) \\
\text { Zhou et al. }{ }^{15}(191)\end{array}$ & $\begin{array}{l}100 \% \text { - } \\
\text { Presence }\end{array}$ & $\begin{array}{c}\text { Huang et al. }{ }^{17}(41) \\
\text { Zhang et al. }{ }^{6}(19) \\
\text { Wang et al. }{ }^{7}(138) \\
\text { Liu et al. }{ }^{10}(78) \\
\text { Liu et al. } .^{9}(109) \\
\text { Ruan et al. } .^{14}(150)\end{array}$ & $\begin{array}{c}\text { Yang et al. }{ }^{19}(52) \\
\text { Zhonghua }^{22} \\
(44672) \\
\text { Guan et al. }{ }^{18} \\
\text { (1099) }\end{array}$ & -3 & $\begin{array}{c}\text { Caramelo et al. }^{12} \\
\text { CRD: OR } 7.8 \\
95 \% \text { Cl } 5.5-10.4\end{array}$ & $\bullet \bullet ०$ & $\begin{array}{c}10 \\
(2 / 12=17 \%) \\
\text { Percentages } \\
\text { COPD, NA }\end{array}$ \\
\hline Cancer & $\begin{array}{c}\text { Caramelo et al. }{ }^{12} \\
\quad(72314)\end{array}$ & $\begin{array}{l}100 \% \text { - } \\
\text { Presence }\end{array}$ & $\begin{array}{l}\text { Huang et al. }{ }^{17}(41) \\
\text { Liu et al. }{ }^{10}(78) \\
\text { Ruan et al. } .^{14}(150) \\
\text { Zhou et al. } .^{15}(191)\end{array}$ & $\begin{array}{l}\text { Zhonghua }^{22} \\
\quad(44672) \\
\text { Guan et al. }^{18} \\
\text { (1099) }\end{array}$ & -3 & $\begin{array}{c}\text { Liu et al. }{ }^{10} \\
\text { Cancer: OR } 6.9 \\
95 \% \text { Cl } 3.4-12.5\end{array}$ & $\bullet \bullet ०$ & $\begin{array}{c}7 \\
(0 / 7)=0 \% \\
\text { Percentages } \\
\text { Malignancy, NA }\end{array}$ \\
\hline $\begin{array}{l}\text { Cardiovascular } \\
\text { disease }\end{array}$ & $\begin{array}{l}\text { Caramelo et al. }{ }^{12} \\
\qquad(72314) \\
\text { Ruan et al. }{ }^{14}(150) \\
\text { Zhou et al. }{ }^{15}(191)\end{array}$ & $\begin{array}{l}100 \% \text { - } \\
\text { Presence }\end{array}$ & $\begin{array}{c}\text { Huang et al. }{ }^{17}(41) \\
\text { Wang et al. }{ }^{7}(138) \\
\text { Liu et al. } .^{9}(109) \\
\text { Zhang et al. }{ }^{6}(19) \\
\text { Liu et al. }{ }^{10}(78) \\
\text { Li et al. } .^{11}(84) \\
\text { Wu et al. } .^{16}(201)\end{array}$ & $\begin{array}{c}\text { Guan et al. }{ }^{18} \\
(1099) \\
\text { Yang et al. } .^{19}(52) \\
\text { Zhonghua }^{22} \\
(44672)\end{array}$ & -4 & $\begin{array}{c}\text { Caramelo et al. }^{12} \\
\text { HTA: OR } 7.4 \\
95 \% \text { Cl } 6.3-8.8 \\
\text { Cardiac: OR } 12.8 \\
95 \% \text { Cl } 10.3-15.9\end{array}$ & $\bullet \bullet$ & $\begin{array}{c}12 \\
(1 / 13)=8 \% \\
\text { Percentages } \\
\text { Cardiac disease, } \\
\text { NA }\end{array}$ \\
\hline Lactate & Liu et al..$^{9}(109)$ & $100 \%-\uparrow$ & 0 & $\begin{array}{c}\text { Yang et al. } .^{19}(52) \\
\text { Wang et al. }{ }^{7}(138)\end{array}$ & -1 & NA & $\bullet ০ ০$ & $\begin{array}{c}3 \\
(0 / 3)=0 \% \\
\text { Median (IQR) } \\
\text { Lactate, } \mathrm{mmol} / \mathrm{L}\end{array}$ \\
\hline $\begin{array}{l}\text { Monocyte } \\
\text { count }\end{array}$ & Li et al. ${ }^{11}(84)$ & $100 \%-\downarrow$ & $\begin{array}{l}\text { Wang et al. }{ }^{7}(138) \\
\text { Wu et al. }{ }^{16}(201)\end{array}$ & 0 & -1 & $\mathrm{NA}$ & $\bullet ০ ০$ & $\begin{array}{c}3 \\
(0 / 3)=0 \% \\
\text { Median }(\mathrm{IQR}) \\
\text { Lymphocite } \\
\text { count / ×109/L }\end{array}$ \\
\hline Creatinine & $\begin{array}{l}\text { Ruan et al. }{ }^{14}(150) \\
\text { Wang et al. }{ }^{7}(138) \\
\text { Zhou et al. }{ }^{15}(191)\end{array}$ & $100 \%-\uparrow$ & $\begin{array}{c}\text { Liu et al. }{ }^{10}(78) \\
\text { Huang et al. }{ }^{17}(41) \\
\text { Liu et al. } .^{9}(109) \\
\text { Wu et al. }{ }^{16}(201)\end{array}$ & $\begin{array}{c}\text { Yang et al. } .^{19}(52) \\
\text { Guan et al. }{ }^{18} \\
\text { (1099) }\end{array}$ & -1 & $\mathrm{NA}$ & $\bullet \circ$ & $\begin{array}{c}5 \\
(4 / 9)=44 \% \\
\text { Median }(\mathrm{IQR}) \\
\text { Creatinin, } \mu \mathrm{mol}\end{array}$ \\
\hline $\begin{array}{l}\text { Composite } \\
\text { abnormal } \\
\text { radiological } \\
\text { findings } \\
\text { (CT-RX) }\end{array}$ & $\begin{array}{l}\text { Li et al. }{ }^{11}(84) \\
\text { Zhou et al. }{ }^{15}(191)\end{array}$ & $100 \%-\uparrow$ & $\begin{array}{l}\text { Wang et al. } .^{7}(138) \\
\text { Zhang et al. }{ }^{6}(19) \\
\text { Huang et al. }{ }^{17}(41)\end{array}$ & $\begin{array}{c}\text { Young et al. } .^{20}(18) \\
\text { Guan et al. } .^{18} \\
(1099)\end{array}$ & -1 & NA & $\bullet \circ ০$ & $\begin{array}{c}0 \\
(6 / 6)=100 \% \\
\text { NA } \\
\text { NA }\end{array}$ \\
\hline $\begin{array}{l}\text { Systolic blood } \\
\text { pressure }\end{array}$ & Huang et al. ${ }^{17}(41)$ & $100 \%-\uparrow$ & $\begin{array}{l}\text { Tian et al. }{ }^{8}(262)^{*} \\
\text { Zhou et al. } .^{15}(191)\end{array}$ & $\begin{array}{l}\text { Yang et al. }{ }^{19}(52) \\
\text { Young et al. }{ }^{20}(18)\end{array}$ & -1 & $\mathrm{NA}$ & $\bullet \circ$ & $\begin{array}{c}2 \\
(2 / 5)=40 \% \\
\text { Mean (SD) } \\
\text { Median (IQR) } \\
\text { Systolic blood } \\
\text { pressure, mmHg }\end{array}$ \\
\hline Platelet count & $\begin{array}{l}\text { Ruan et al. }{ }^{14}(150) \\
\text { Zhou et al. }{ }^{15}(191)\end{array}$ & $100 \%-\downarrow$ & $\begin{array}{c}\text { Wang et al. }{ }^{7}(138) \\
\text { Liu et al. }{ }^{10}(78) \\
\text { Wu et al. } .^{16}(201)\end{array}$ & $\begin{array}{c}\text { Yang et al. } .^{19}(52) \\
\text { Tang et al. }{ }^{13}(183) \\
\text { Young et al. } .^{20}(18) \\
\text { Guan et al. }{ }^{18} \\
\text { (1099) } \\
\text { Huang et al. } .^{17}(41)\end{array}$ & -1 & $\mathrm{NA}$ & $\bullet \circ$ & $\begin{array}{c}5 \\
(5 / 10)=50 \% \\
\text { Median (IQR) } \\
\text { Platelet count / } \\
\times 109 / \mathrm{L}\end{array}$ \\
\hline AST & $\begin{array}{l}\text { Wang et al. }{ }^{7}(138) \\
\text { Wu et al. }{ }^{.6}(201)\end{array}$ & $100 \%-\uparrow$ & $\begin{array}{c}\text { Liu et al. }{ }^{10}(78) \\
\text { Liu et al. } .^{9}(109) \\
\text { Ruan et al. } .^{14}(150)\end{array}$ & $\begin{array}{c}\text { Guan et al. }{ }^{18} \\
\text { (1099) } \\
\text { Huang et al. }{ }^{17}(41)\end{array}$ & -1 & NA & $\bullet ০ ০$ & $\begin{array}{c}6 \\
(1 / 7)=14 \% \\
\text { Median (IQR) } \\
\text { Aspartate } \\
\text { aminotransferase, } \\
\text { UL }\end{array}$ \\
\hline
\end{tabular}

Continue 
Table. Summary of risk factors associated with covid-19 severity and evaluation of reporting consistency. Continuation

\begin{tabular}{|c|c|c|c|c|c|c|c|c|}
\hline ALT & $\begin{array}{l}\text { Wang et al. }{ }^{7}(138) \\
\text { Huang et al. }{ }^{17}(41) \\
\text { Zhou et al. } .^{15}(191)\end{array}$ & $100 \%-\uparrow$ & $\begin{array}{l}\text { Liu et al. }{ }^{10}(78) \\
\text { Liu et al. } .^{9}(109) \\
\text { Ruan et al. }{ }^{14}(150) \\
\text { Wu et al. } .^{16}(201)\end{array}$ & $\begin{array}{l}\text { Guan et al. }{ }^{18} \\
\text { (1099) }\end{array}$ & -1 & $\mathrm{NA}$ & $\bullet$ & $\begin{array}{c}6 \\
(2 / 8)=25 \% \\
\text { Median (IQR) } \\
\text { Alanine } \\
\text { aminotransferase, } \\
\text { UL }\end{array}$ \\
\hline Expectoration & Li et al. ${ }^{11}(84)$ & $\begin{array}{c}100 \%- \\
\text { Presence }\end{array}$ & 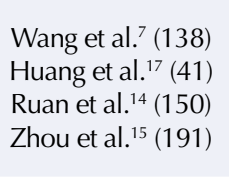 & $\begin{array}{l}\text { Guan et al. }{ }^{18} \\
(1099)\end{array}$ & -3 & NA & $\bullet$ & $\begin{array}{c}6 \\
(0 / 6)=0 \% \\
\text { Percentages } \\
\text { Sputum, NA }\end{array}$ \\
\hline Cough & $\begin{array}{c}\text { Li et al. }{ }^{11}(84) \\
\text { Zhang et al. }{ }^{6}(19)\end{array}$ & $\begin{array}{c}100 \%- \\
\text { Presence }\end{array}$ & $\begin{array}{l}\text { Huang et al. }{ }^{17}(41) \\
\text { Tian et al. }{ }^{8}(262) \\
\text { Wang et al. }{ }^{7}(138) \\
\text { Liu et al. }{ }^{10}(78) \\
\text { Liu et al. } .^{9}(109) \\
\text { Ruan et al. } .^{14}(150) \\
\text { Zhou et al. } .^{15}(191) \\
\text { Wu et al. } .^{16}(201)\end{array}$ & $\begin{array}{l}\text { Yang et al. } .^{19}(52) \\
\text { Guan et al. }{ }^{18} \\
\quad(1099) \\
\text { Young et al. }{ }^{20}(18)\end{array}$ & -6 & $\mathrm{NA}$ & $\bullet$ & $\begin{array}{l}11 \\
(2 / 13)=15 \% \\
\text { Percentages } \\
\text { Cough, NA }\end{array}$ \\
\hline Fatigue & Liu et al. ${ }^{9}(109)$ & $\begin{array}{c}100 \% \text { - } \\
\text { Presence }\end{array}$ & $\begin{array}{l}\text { Huang et al. } .^{17}(41) \\
\text { Zhang et al. }{ }^{6}(19) \\
\text { Tian et al. }{ }^{8}(262) \\
\text { Wang et al. }{ }^{7}(138) \\
\text { Ruan et al. }{ }^{14}(150) \\
\text { Wu et al. } .^{16}(201) \\
\text { Zhou et al. }{ }^{15}(191)\end{array}$ & $\begin{array}{c}\text { Guan et al. }{ }^{18} \\
(1099)\end{array}$ & -6 & $\mathrm{NA}$ & $\bullet$ & $\begin{array}{c}5 \\
(2 / 7)=29 \% \\
\text { Percentages } \\
\text { Fatigue, NA }\end{array}$ \\
\hline Male gender & $\begin{array}{c}\text { Tang et al. }{ }^{13}(183) \\
\text { Caramelo et al. }{ }^{12} \\
\quad(72314)\end{array}$ & $\begin{array}{c}100 \%- \\
\text { Presence }\end{array}$ & $\begin{array}{l}\text { Huang et al. } .^{17}(41) \\
\text { Zhang et al. } .^{6}(19) \\
\text { Wang et al. } .^{7}(138) \\
\text { Tian et al. }{ }^{8}(262) \\
\text { Liu et al. } .^{9}(109) \\
\text { Li et al. }{ }^{11}(84) \\
\text { Ruan et al. }{ }^{14}(150) \\
\text { Liu et al. } .^{10}(78) \\
\text { Zhou et al. }{ }^{15}(191) \\
\text { Wu et al. }{ }^{16}(201)\end{array}$ & 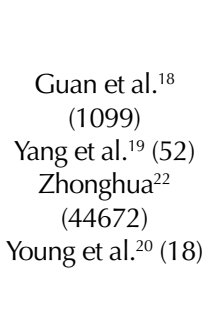 & -8 & $\mathrm{NA}$ & $\bullet$ & $\begin{array}{c}15 \\
(1 / 16)=6 \% \\
\text { Percentages } \\
\text { Male, NA }\end{array}$ \\
\hline
\end{tabular}

HTA: hypertension; CRD: chronic respiratory disease

* Data provided directly by the authors of the publication.

performed multivariate analysis adjusting for confounding factors. Sixteen of the studies reported laboratory-confirmed cases of covid-19 and one reported clinically diagnosed cases. There were 60 risk factors identified for COVID-19 severity (Table). Of these, 7 were considered of high, 40 of medium and 13 of low consistency. Increasing values of age, D-dimer, C-reactive protein, sequential organ failure assessment (SOFA) score and body temperature while decreasing albumin, and a history of diabetes were the risk factors with the highest consistency as predictors for covid-19 severity. Additionally, elevated values of white blood cells count, procalcitonin, lactate dehydrogenase, cardiac troponins, prothrombin time, interleukin-6, serum ferritin, neutrophils count, creatine kinase MB, CURB-65 score with decreased lymphocyte count, and dyspnea were classified as medium consistency risk factors with at least a positive difference of two studies reporting a statistically significant difference between non-severe and CSF groups. There was high heterogeneity in the definition of CSF, ranging from the need for supplemental oxygen to the development of acute respiratory distress syndrome (ARDS), ICU admission and death. In terms of risk factor heterogeneity, $40 \%$ of factors presented a value of zero with an overall median of $14 \%$ (IQR $=0-25)$. Nevertheless, when considering only the remaining $60 \%$ variables, the mean heterogeneity value was $28.5 \%(\mathrm{SD}=19.6)$.

\section{DISCUSSION}

The results from this review are consistent with current analyses considering age and comorbidities the most important risk factors for covid-19 disease severity. However, 
our findings also suggest that diabetes is one of the most critical comorbidities in terms of disease severity. Diabetes has been previously associated with other respiratory virus disease severities in cross national samples ${ }^{5}$. This might be explained by the immunosuppressive effects of hyperglycaemia ${ }^{5}$ and could also explain why patients that develop ARDS due to covid-19 were found to have statistically significant higher glucose levels (Table). This finding has important implications given the high global prevalence of diabetes. When considering the heterogeneity of reported factors across studies, $60 \%$ presented some level of heterogeneity, which indicates that there is a need for higher reporting consistency in future research looking at the risk factors for covid-19 disease severity.

Some limitations should be considered when interpreting these findings. Most of the selected studies were conducted in China (and one in Singapore), limiting the external validity of risk factors for other countries. As we used a composite index of severity, the relevance of these factors varies according to the natural history of the disease with some factors, such as body temperature and neutrophils count (and their value directions -higher/lower) being more relevant for different stages. A limitation of this study is the rapid growth of knowledge about covid-19. Therefore, the results of the present review might vary as the scientific understanding of covid-19 progresses. Additionally, two of the 17 studies reviewed were pre-prints (neither published, nor peer-reviewed). Despite this, the wide range of risk factors identified across 17 publications can guide future research to rapidly validate the present results on cross-national samples.

Given that the burden to the health system due to covid-19 is a determinant of the disease severity (Table), the results from this review can support clinical and public health initiatives to target populations and patients that are at most risk while further evidence is generated. Additionally, the present review provides researchers with a rapid reference on reported clinical and demographical factors in order to increase the comparability of results and further decrease uncertainties regarding the covid-19 severity. This can support clinical management decisions and the design of strategies to inform the general public about important risk factors for covid-19 severity, For instance, when communicating who is most at risk for the disease, instead of making a broad generalization such as "increasing age and underlying health conditions," messaging that communicates risk factors should at a minimum include: age > 50, diabetes, smoking, respiratory disease, cancer and cardiovascular disease. Additionally, for patients that are isolated outside a healthcare institution either due to clinical suspicion or confirmed mild case, specific factors such as shortness of breath and chest pain could be communicated as triggers for seeking care. Factors such as fatigue, cough and expectoration have low consistency for predicting disease severity and by themselves should not be relied upon for clinical assessment. We expect that the results of this brief review can support government and medical strategies in response to the pandemic.

\section{REFERENCES}

1. World Health Organization. Coronavirus disease 2019 (COVID-19) Situation Report - 78. Geneva: WHO; 2020.

2. Lipsitch M, Swerdlow DL, Finelli L. Defining the epidemiology of Covid-19: studies needed. N Engl J Med. 2020;382(13):1194-6. https://doi.org/10.1056/NEJMp2002125

3. Murad MH, Asi N, Alsawas M, Alahdab F. New evidence pyramid. Evid Based Med. 2016;21(4):125-7. https://doi.org/10.1136/ebmed-2016-110401

4. Guan WJ, Ni ZY, Hu Y, Liang WH, Ou CQ, He JX, et al. Clinical characteristics of Coronavirus Disease 2019 in China. N Engl J Med. 2020;382:1708-20. Chinese translation https://doi.org/10.1056/NEJMoa2002032 
5. Hulme KD, Gallo LA, Short KR. Influenza Virus and glycemic variability in diabetes: a killer combination? Front Microbiol. 2017;8:861. https://doi.org/10.3389/fmicb.2017.00861

6. Zhang JJ, Dong X, Cao YY, Yuan YD, Yang YB, Yan YQ, et al. Clinical characteristics of 140 patients infected with SARS-CoV-2 in Wuhan, China [published online ahead of print, 2020 Feb 19]. Allergy. 2020;10.1111/all. https://doi.org/10.1111/all.14238

7. Wang D, Hu B, Hu C, Zhu F, Liu X, Zhang J, et al. Clinical characteristics of 138 hospitalized patients with 2019 novel coronavirus-Infected Pneumonia in Wuhan, China. JAMA. 2020;323(11):1061-9. https://doi.org/10.1001/jama.2020.1585

8. Tian S, Hu N, Lou J, Chen K, Kang X, Xiang Z, et al. Characteristics of COVID-19 infection in Beijing. J Infect. 2020;80(4):401-6. https://doi.org/10.1016/j.jinf.2020.02.018

9. Liu Y, Sun W, Li J, Chen L, Wang Y, Zhang L, et al. Clinical features and progression of acute respiratory distress syndrome in coronavirus disease 2019 [preprint]. medRxiv. 2020. https://doi.org/10.1101/2020.02.17.20024166

10. Liu W, Tao ZW, Lei W, Ming-Li Y, Kui L, Ling Z, et al. Analysis of factors associated with disease outcomes in hospitalized patients with 2019 novel coronavirus disease [published online ahead of print, 2020 Feb 28]. Chin Med J (Engl). 2020. https://doi.org/10.1097/CM9.0000000000000775

11. Li K, Wu J, Wu F, Guo D, Chen L, Fang Z, et al. The clinical and chest CT features associated with severe and critical COVID-19 Pneumonia [published online ahead of print, 2020 Feb 29]. Invest Radiol. 2020. https://doi.org/10.1097/RLI.0000000000000672

12. Caramelo F, Ferreira N, Oliveiros B. Estimation of risk factors for COVID-19 mortality: preliminary results [preprint]. medRxiv. 2020. https://doi.org/10.1101/2020.02.24.20027268

13. Tang N, Li D, Wang X, Sun Z. Abnormal coagulation parameters are associated with poor prognosis in patients with novel coronavirus pneumonia. J Thromb Haemost. 2020;18(4):844-7. https://doi.org/10.1111/jth.14768

14. Ruan Q, Yang K, Wang W, Jiang L, Song J. Clinical predictors of mortality due to COVID-19 based on an analysis of data of 150 patients from Wuhan, China [published online ahead of print, 2020 Mar 3]. Intensive Care Med. 2020. https://doi.org/10.1007/s00134-020-05991-x

15. Zhou F, Yu T, Du R, Fan G, Liu Y, Liu Z, et al. Clinical course and risk factors for mortality of adult inpatients with COVID-19 in Wuhan, China: a retrospective cohort study. Lancet. 2020;395(10229):1054-62. https://doi.org/10.1016/S0140-6736(20)30566-3

16. Wu C, Chen X, Cai Y, Xia J, Zhou X, Xu S, et al. Risk factors associated with acute respiratory distress syndrome and death in patients with Coronavirus Disease 2019 Pneumonia in Wuhan, China [published online ahead of print, 2020 Mar 13]. JAMA Intern Med. 2020;e200994. https://doi.org/10.1001/jamainternmed.2020.0994

17. Huang C, Wang Y, Li X, Ren L, Zhao J, Hu Y, et al. Clinical features of patients infected with 2019 novel coronavirus in Wuhan, China. Lancet. 2020;395(10223):497-506. https://doi.org/10.1016/S0140-6736(20)30183-5

18. Guan WJ, Ni ZY, Hu Y, Liang WH, Ou CQ, He JX, et al; China Medical Treatment Expert Group for Covid-19. Clinical characteristics of Coronavirus Disease 2019 in China. N Engl J Med. 2020. Chinese translation. https://doi.org/10.1056/NEJMoa2002032

19. Yang X, Yu Y, Xu J, Shu H, Xia J, Liu H, et al. Clinical course and outcomes of critically ill patients with SARS-CoV-2 pneumonia in Wuhan, China: a single-centered, retrospective, observational study [published online ahead of print, 2020 Feb 24]. Lancet Respir Med. 2020. https://doi.org/10.1016/S2213-2600(20)30079-5

20. Young BE, Ong SWX, Kalimuddin S, Low JG, Tan SY, Loh J, et al. Epidemiologic features and clinical course of patients infected with SARS-CoV-2 in Singapore. JAMA. 2020;323(15):1488-94. https://doi.org/10.1001/jama.2020.3204

21. Ji Y, Ma Z, Peppelenbosch MP, Pan Q. Potential association between COVID-19 mortality and health-care resource availability. Lancet Glob Health. 2020;8(4):e480. https://doi.org/10.1016/S2214-109X(20)30068-1 
22. Novel Coronavirus Pneumonia Emergency Response Epidemiology Team. [The epidemiological characteristics of an outbreak of 2019 Novel Coronavirus Diseases (COVID-19) - China, 2020]. Zhonghua Liu Xing Bing Xue Za Zhi. 2020;41(2):145-51 Chinese. https://doi.org/10.3760/cma.j.issn.0254-6450.2020.02.003

Funding: J.E. Rod is the recipient of an ATN-LATAM QUT Postgraduate Research Scholarship and a QUT HDR Tuition Fee Sponsorship. Dr Oscar Oviedo-Trespalacios is the recipient of an Australian Research Council Discovery Early Career Researcher Award [DE200101079] funded by the Australian Government

Authors' Contribution: Study design: JER, OOT, JCR. Data collection, analysis and interpretation: JER, OOT, JCR. Writing of the manuscript and approval of the final version: JER, OOT, JCR. Public responsibility for the content of the article: JER, OOT, JCR.

Acknowledgements: To the contribution of a team of medical professionals that conducted part of the data extraction: Laura Acosta Camargo, Moises Molina Fuentes, John David Rodriguez and Juan Carlos Pantoja.

Conflict of Interest: The authors declare no conflict of interest. 